\title{
Synthesis of Polypyrrole Using Ferric Chloride (FeCl 3 ) as Oxidant Together with Some Dopants for Use in Gas Sensors
}

\author{
Hemant K. Chitte ${ }^{*}$, Narendra V. Bhat ${ }^{2}$, Vasant E. Walunj ${ }^{2}$, Ganesh N. Shinde ${ }^{3}$ \\ ${ }^{1}$ Department of Physics, Dnyansadhana College, Near Eternity Mall, Thane, India \\ ${ }^{2}$ Bombay Textile Research Association, Mumbai, India \\ ${ }^{3}$ Department of Physics, Indira Gandhi College, Cidco Colony, New Nanded, India \\ E-mail:hkchitte@yahoo.co.in \\ Received May 3, 2011; revised June 1, 2011; accepted June 7, 2011
}

\begin{abstract}
Polypyrrole (Ppy) was synthesized using Ferric Chloride $\left(\mathrm{FeCl}_{3}\right)$ as oxidant. The ratio of monomer to oxidant was 1:2.4. The polymerization was carried out at $5^{\circ} \mathrm{C}$. Attempts were made to increase the electrical conductivity by using various dopants viz. Lithium per Chlorate $\left(\mathrm{LiClO}_{4}\right)$, para-Toluene Sulfonate (p-TS) and Napthalene Sulfonic acid (NSA). The materials were characterized using FTIR, X-Ray diffraction and SEM. The electrical conductivity was measured by two probe method and was found to be in the range of $10^{-3}$ to $10^{-2} \mathrm{~S} / \mathrm{cm}$. Thin films of these preparations were casted on the interdigited electrodes to study the detection of gases such as ammonia and LPG. It was found that for the pure Ppy and one doped with $\mathrm{LiClO}_{4}$, when ammonia gas was allowed to flow in, there was a sudden increase in the current, which decreased rapidly when gas was stopped. This behavior is in contradiction to that observed in earlier investigations where Ppy was prepared by electrochemical method. However when Ppy doped with p-TS and NSA were used, the trend was reversed. Similarly it was noted that for pure Ppy and one doped with $\mathrm{LiClO}_{4}$, p-TS and NSA there was an increase in the current when LPG gas was brought in. However the response to LPG was somewhat low as compared ammonia gas.
\end{abstract}

Keywords: Polypyrrole, Structure, Doping, Gas Sensor, Ammonia

\section{Introduction}

Electroactive conducting polymers have assumed a great importance in several technologies such as Display devices, Solar cells, Gas sensors and Actuators [1-3]. Their ease of processing together with their chemically tunable properties makes them useful in electro-mechanical devices. Delocalized electronic states combined with the restriction on the extent of delocalization makes most of the conductive polymers behave like p-type semiconductors. As these polymers are Redox - active their conductivity can be changed by means of doping/dedoping. A great number of sensing applications are designed by exploiting the very nature of conducting polymers.

Polypyrrole, in the form of films, have been used for sensors for detection of various gases and volatile organic compounds [4-7]. Polypyrrole sensors are sensitive with good response to these gases, showing larger re- sponse to polar than non-polar compounds.

Reliable sensing of Ammonia $\left(\mathrm{NH}_{3}\right), \mathrm{LPG}$ and volatile organic compounds (VOC) are required in many applications like detection of leaks, explosives, fertilizer industries, compressors of air conditioners, breath analysis for medical diagnosis etc. Further, its high toxicity also warrants a rapid detection at very low concentrations [8].

The physical properties of conducting polymers strongly depend on the type of dopant and the doping levels. The doping levels can be easily changed by chemical reactions at room temperatures and this provides a simple technique to facilitate detection of several gases. Most of the conducting polymers are doped/undoped by redox reactions. When such conducting polymeric material is brought in contact with either a gas or a liquid, transfer of electrons from or to the analyte takes place. Electron transferring can cause the change in resistance and work function of the sensing material. When 
Ppy is exposed to some gases, redox reaction can take place. Gases such as $\mathrm{NO}_{2}$ and $\mathrm{I}_{2}$ which are electron acceptors can remove electrons from the aromatic ring of polypyrrole. When this occurs, for a p-type conductive polymer, the doping level as well as electric conductance of the conductive polymers is enhanced. On the other hand an electron donating gas, such as ammonia, reacts with the Ppy, the electrical conductance falls down sharply. However when desorption of the gas occurs the process can be reversed. The following reaction is involved in the process [9].

$$
\mathrm{Ppy}^{+}+\mathrm{NH}_{3} \rightarrow \mathrm{Ppy}^{\mathrm{O}}+\mathrm{NH}_{3}{ }^{++} \quad \text { absorption }
$$$$
\mathrm{Ppy}^{\mathrm{o}}+\ddot{\mathrm{N}} \mathrm{H}_{3} \rightarrow \mathrm{Ppy}^{+}+\ddot{\mathrm{N}} \mathrm{H}_{3} \quad \text { desorption }
$$

In spite of such advances, the results obtained for many gases may differ from this generalized postulate of detection mechanism. For example work reported in thin polycarboazole film sensors shows increase in conductance when the electron donating gas has been used [10].

In the present paper we report preparation of polypyrrole in pure form and by using dopants such as $\mathrm{LiClO}_{4}$, p-TS and NSA. The structure and morphology of these materials has been investigated using the methods of FTIR, X-Ray diffraction and SEM. The electrical conductivity has been measured by two probe method. The response of these materials, when exposed to ammonia and LPG is being reported.

\section{Experimental Methods and Preparations}

Pyrrole (Sisco Research laboratory, 99\% pure) was distilled before use. All other reagents and solvents obtained from SDL were of reagent grade and were used as received. All solutions were prepared using distilled water. All reactions were conducted at a temperature of $5^{\circ} \mathrm{C}$ [11]. The solution of the oxidizing agent, $\mathrm{FeCl}_{3}$, was prepared using distilled water and was used in the ratio of 1:2.4 (monomer: oxidant) [12]. Dopants were mixed with Pyrrole solution $(10 \% \mathrm{w} / \mathrm{w})$ and stirred for $30 \mathrm{~min}$ utes for proper mixing and then the oxidant solution was added slowly.

The Polypyrrole was prepared by chemical polymerization method. $1 \mathrm{M}$ Pyrrole solution was prepared using distilled water and then mixed with oxidizing agents in the ratio mentioned above, slowly under constant stirring for 30 minutes. Then the polymerization was conducted for 4 hours under constant stirring. This preparation was kept unagitated for 24 hours so that Ppy powder settled down. The Polypyrrole powder was filtered out under vacuum and washed with distilled water several times to remove any impurities present. The Polypyrrole was dried for 2 days at room temperature.

The chemical bonding was analyzed using FTIR spectroscopy by FTIR spectrometer model Perkin-Elmer Ltd. system 2000 using $\mathrm{KBr}$ pellets. The Polypyrrole was characterized by XRD using PANalytical (Philips), model XpertPro. The XRD patterns were recorded between $2 \theta=10^{\circ}$ to $40^{\circ}$. The X-ray diffractometer uses $\mathrm{CuK} \alpha$ radiation of $\lambda=1.5418 \mathrm{~A}^{0}$ generated at $40 \mathrm{kV} / 20 \mathrm{ma}$.

The morphology of Polypyrrole was examined using Scanning Electron Microscope (SEM) JEOL make JSM - 5400 model. The Polypyrrole was tested for conductivity by preparing its pellets of area of cross section of 1 sq.cm. and $1 \mathrm{~mm}$. thickness. The conductivity was tested by two probe method at room temperature and various temperatures up to $70^{\circ} \mathrm{C}$ in the voltage range from 0 to $12 \mathrm{~V}$.

In order to measure the gas response, interdigited electrodes, separated by $1 \mathrm{~mm}$. from all sides, were prepared on a printed circuit board (PCB) and the slurry of Polypyrrole powder prepared with distilled water was spread over it uniformly and dried for 24 hours under vacuum at room temperature. A specially prepared gas chamber was used in which the PCB was fitted firmly. Various gases were passed through the chamber at room temperature with Nitrogen gas as a carrier. The current was recorded, at a constant voltage, for every 15 seconds for the total time of 3 minutes. The flow of the gas was stopped and the desorption was also recorded for interval of every 10 seconds till current recovered up to $90 \%$ of its original value. The gas response was recorded continuously for 3 cycles.

\section{Result and Discussions}

\subsection{Characterization of Ppy}

\subsubsection{FTIR Spectroscopy}

The Polypyrrole powders prepared in different ways were analyzed by FTIR. FTIR spectra showed the main characteristic peaks at $788 \mathrm{~cm}^{-1}$ corresponding to C-N bond, $1291 \mathrm{~cm}^{-1}$ corresponding to $\mathrm{C}-\mathrm{H}$ deformation, $1540 \mathrm{~cm}^{-1}$ and $1467 \mathrm{~cm}^{-1}$ corresponding to the fundamental vibrations of polypyrrole ring, The peak at $1640 \mathrm{~cm}^{-1}$ corresponding to $\mathrm{C}=\mathrm{C}$. The peak at $3411 \mathrm{~cm}^{-1}$ corresponds to the N-H bond $[13,14]$. These peaks were observed in the present work for preparations using $\mathrm{FeCl}_{3}$ as oxidants and various dopants such as $\mathrm{LiClO}_{4}$, p-TS and NSA (Figure 1). This agrees well with the ones available the literature, confirming the formation of Polypyrrole $[13,15]$.

\subsubsection{X-Ray Diffraction Analysis}

A typical $\mathrm{X}$ ray diffraction pattern for polypyrrole prepared using $\mathrm{FeCl}_{3}$ as oxidant is shown in Figure 2. The XRD patterns for samples using the dopants also show 


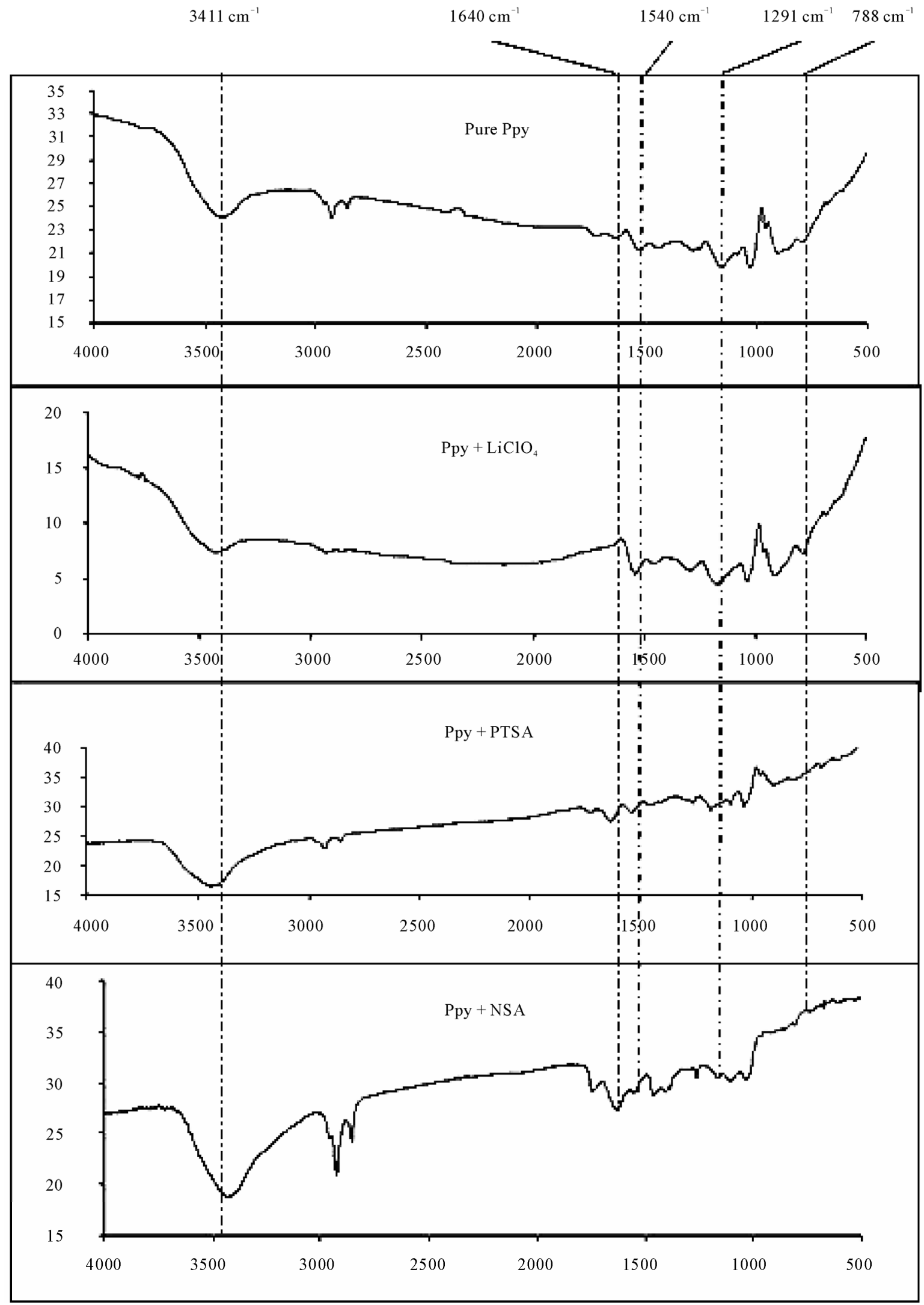

Figure 1. FTIR of Polypyrrole using $\mathrm{FeCl}_{3}$, and dopants $\mathrm{P}$-TS, NSA, $\mathrm{LiClO}_{4}$. 


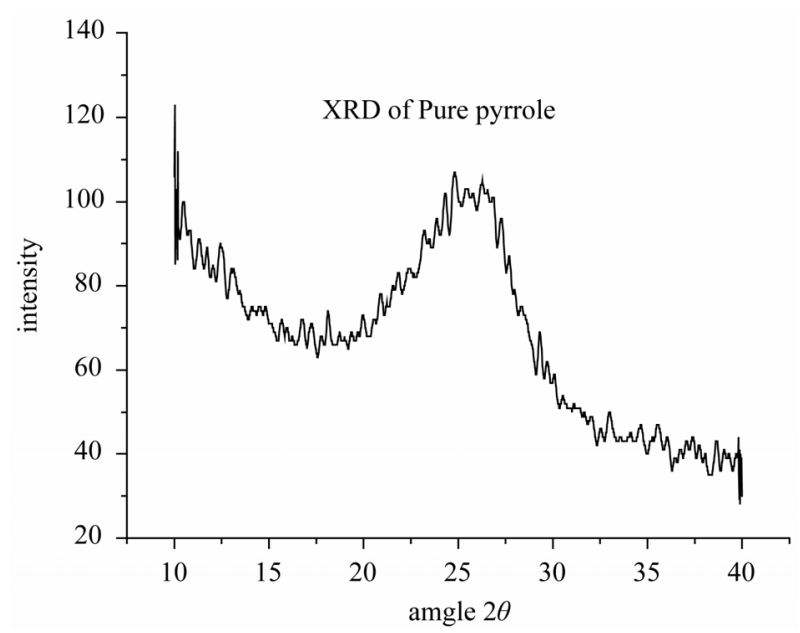

Figure 2. $\mathrm{X}$ ray diffraction pattern for polypyrrole prepared using $\mathrm{FeCl}_{3}$ as oxidant.

broad peaks in the region $15^{\circ}<2 \theta<30^{\circ}$ revealing that the resulting polypyrrole powders are amorphous in nature. This agrees well with the structure reported in literature [16]. Such broad peak usually indicates short range arrangement of chains. The half width of this peak was measured for all the four samples and their values are given in Table 1. The half widths were measured from the diffraction curve from the angles higher than the peak positions and assuming Gaussian distribution for the low angle part of the diffraction curve.

However the diffraction peaks centered at around $26^{\circ}$ shows some displacement when we go from pure pyrrole to the doped ones. In general the doping leads to shift of the peaks toward the higher angle by about $1^{\circ}$. This indicates that the inter planer spacing decreases with the additon of dopants. This would make the chains come closer as also the interaction of dopants stronger leading to higher overlap of bonds. This can cause increase in the conduction which has indeed been observed in the present investigation. In addition it was noted that the diffraction profile is highly asymmetrical and there is considerable broadening towards lower angles from the peak positions.

\subsubsection{Morphology Using SEM}

The morphological features of polypyrrole synthesis che- mically and electrochemically have revealed that mostly the growth is in the globular form but changes some time due to dopant molecules. Typical SEM images of different Polypyrrole preparations are shown in Figure 3. All the photographs show a globular structure [16]. It can be seen from Figure 3(a) that when polymerization was done with $\mathrm{FeCl}_{3}$ the average size of globules was found to be $0.59 \mu \mathrm{m}$. The individual granules observed were nearly spherical and have a close packing. It seems that such spherulites are growing one over the other and forming a continuous structure. The sizes of these spherulites are varying from $0.4 \mu \mathrm{m}$ to $0.9 \mu \mathrm{m}$. When dopants were used during the polymerization with $\mathrm{FeCl}_{3}$, the sizes of the granules were found to be different. With Li$\mathrm{ClO}_{4}$ the average size was found to be $0.61 \mu \mathrm{m}$, with variation between $0.4 \mu \mathrm{m}$ to $0.8 \mu \mathrm{m}$. When $\mathrm{p}$-TS was used as a dopant, there was a considerable reduction in the size amounting to $0.30 \mu \mathrm{m}$. More over the morphological feature was spongy in nature and there was considerable difficulty in distinguishing the granules from each other. This shows that a much closed packed structure is formed and this fact supports our earlier conclusion on the basis of $\mathrm{X}$ ray diffraction. When NSA was used as dopant it was observed that average globular size is $0.85 \mu \mathrm{m}$ which is rather large in comparison to other preparations. Such morphological features are considered to be good for gas sensing applications.

\subsection{I-V Characteristics}

Typical plots of I vs. V for polypyrrole prepared using $\mathrm{FeCl}_{3}$ as oxidant and $\mathrm{LiClO}_{4}$, p-TS and NSA as dopants are given in Figure 4. Nearly linear relationship of the I-V curve was noted. It was observed that the electrical conductivity of polypyrrole increased when dopants such as $\mathrm{LiClO}_{4}, \mathrm{p}$-TS and NSA were used with $\mathrm{FeCl}_{3}$ as oxidant the conductivities were found in the range of $10^{-3} \mathrm{~S} / \mathrm{cm}$ to $10^{-2} \mathrm{~S} / \mathrm{cm}$.

The temperature dependence of the electrical conductivity of the polypyrrole, prepared with $\mathrm{FeCl}_{3}$ and their doped varieties were studied at various temperatures from $30^{\circ} \mathrm{C}$ up to $70^{\circ} \mathrm{C}$. It was observed that the electrical conductivity decreased gradually for all the samples. This behavior resembles the metallic conductors. It may

Table 1. Half widths, Globular sizes and conductivities of polypyrrole prepared using different dopants.

\begin{tabular}{ccccc}
\hline Sample & X ray peak position & X ray Half width in degrees & SEM Size of Globule & Conductivity at Room Temp. \\
\hline Pure Polypyrrole & $25.63^{\circ}$ & $5.0^{\circ}$ & $0.59 \mu \mathrm{m}$ & $2.14 \times 10^{-3}$ \\
Polypyrrole with $\mathrm{LiClO}_{4}$ & $25.6^{\circ}$ & $5.0^{\circ}$ & $0.61 \mu \mathrm{m}$ & $28.74 \times 10^{-3}$ \\
Polypyrrole with p-TS & $25.3^{\circ}$ & $7.0^{\circ}$ & $0.30 \mu \mathrm{m}$ & $51.00 \times 10^{-3}$ \\
Polypyrrole with NSA & $26.2^{\circ}$ & $5.4^{\circ}$ & $0.85 \mu \mathrm{m}$ & $19.15 \times 10^{-3}$ \\
\hline
\end{tabular}




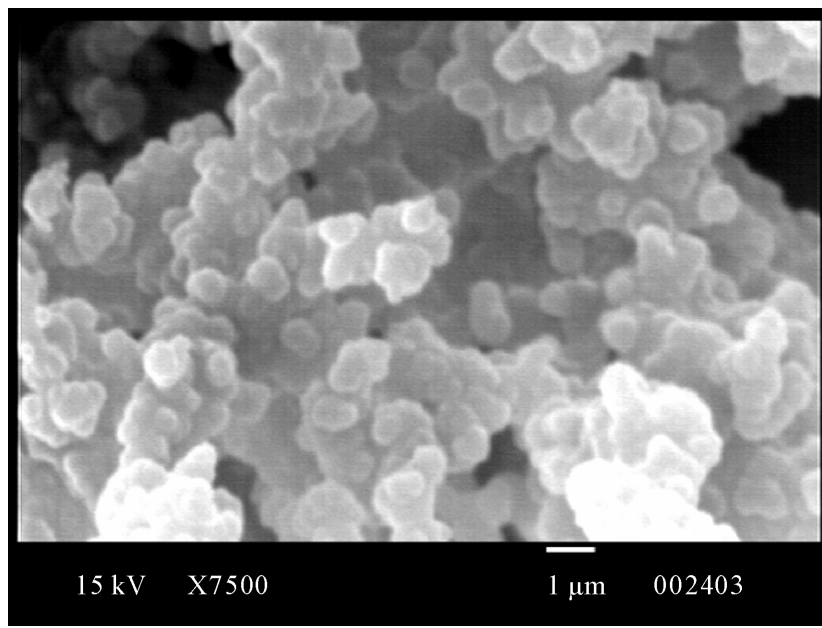

(a)

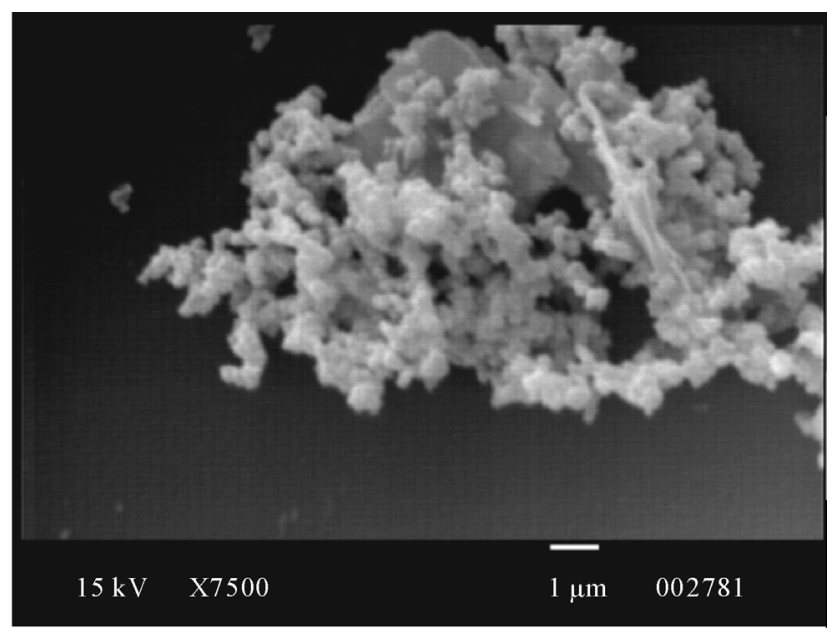

(c)

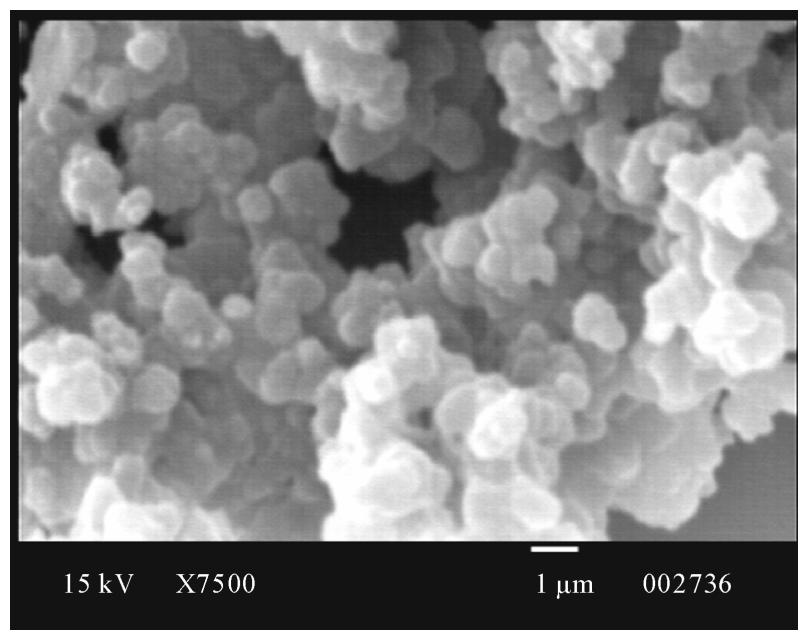

(b)

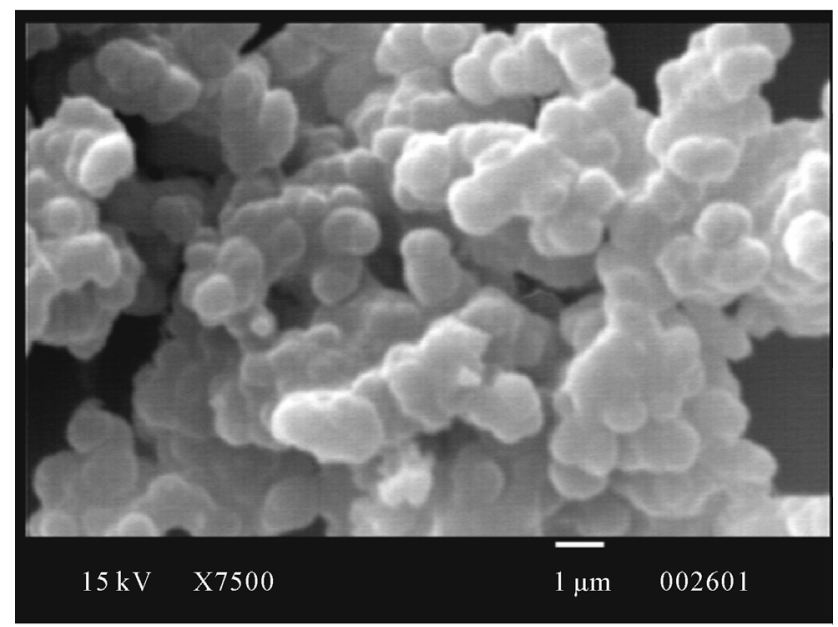

(d)

Figure 3. (a) SEM of Ppy using $\mathrm{FeCl}_{3}$ as an oxidant, prepared by our method; (b) SEM of Ppy using $\mathrm{FeCl}_{3}$ as an oxidant and $\mathrm{LiClO}_{4}$ as dopant, prepared by our method; (c) SEM of Ppy using $\mathrm{FeCl}_{3}$ as an oxidant and P-TS as dopant prepared by our method; (d) SEM of Polypyrrole using $\mathrm{FeCl}_{3}$ as an oxidant and NSA as dopant, prepared by our method.

be mentioned that pure pyrrole is more like insulator but behaves like semiconductor in the doped form. The mechanism of conduction is supposed to be by polarons and bipolarons formation due to the dopant molecules [15]. The mechanism of polaron formation is illustrated in Figure 5. In case of polypyrrole the absence of electron in the chain leads to formation of holes i.e. p-type conduction. Thus addition of dopants leads to the modification of energy levels as shown in Figure 5.

Additional energy bands are formed above the valence band and just below the conduction band. This reduces the energy gap and the doping leads to semiconduction. Thus it is expected that increasing temperature will lead to increase of energy for an electron in the valance band which jumps to conduction band and hence the conductivity should increase with the temperature. However the present studies revealed that the materials do not behave like semiconductor but like metals where the conductivity is decreasing with temperature [17]. Such behavior can be caused due to the large number of intermediate energy states in the energy gap region. In the present studies the doping level was $10 \%$ which is comparatively high and leads to overlap of a large number of energy states. In addition the size of the dopants ions $\mathrm{ClO}_{4}$ and $\mathrm{SO}_{3}^{-}$is quite large and overlap of energy states can occur. Thus the temperature dependence can be understood. The conductivities change in the order p-TS $>\mathrm{LiClO}_{4}>$ NSA > pure.

Of all the samples studied in the present investigation polypyrrole doped with p-TS was found to have maximum conductivity. In addition it was noted that the conductivity does not change much with temperature for 


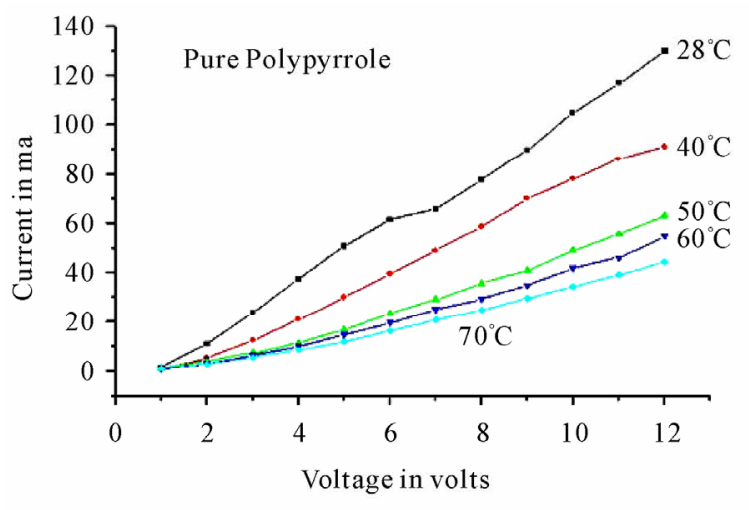

(a)

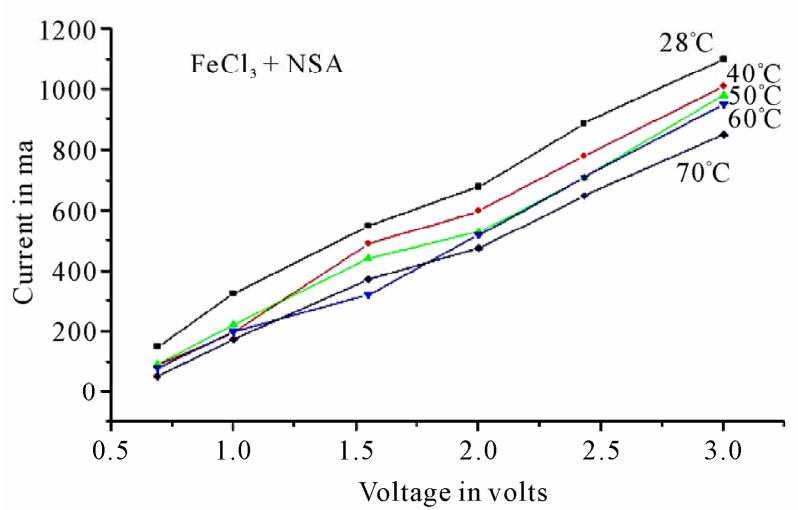

(c)

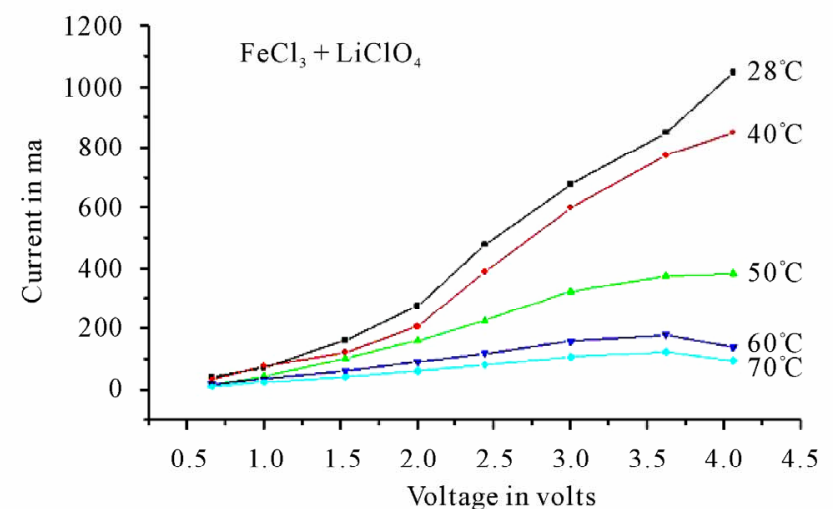

(b)

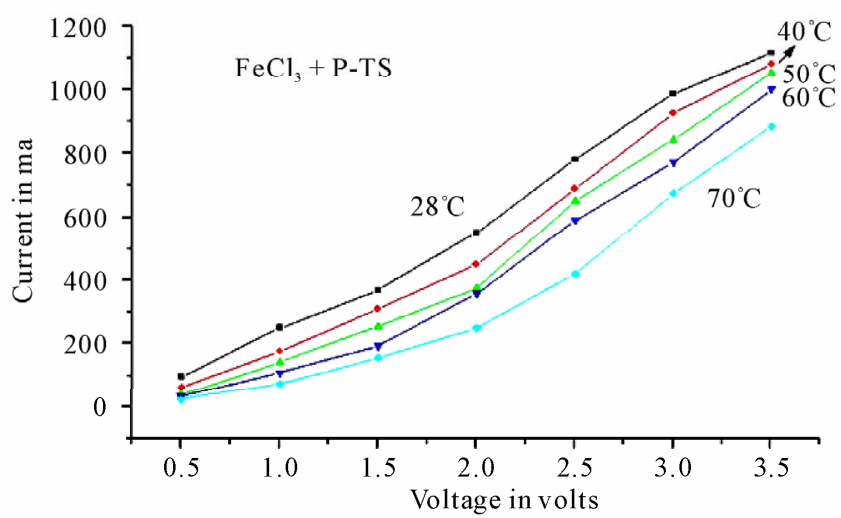

(d)

Figure 4. I vs V characteristic of Polypyrrole at various temperatures prepared using (a) $\mathrm{FeCl}_{3}$, (b) $\mathrm{LiClO}_{4}$ (c) $\mathrm{NSA}_{\text {(d) }}$ - -TS.

NSA and p-TS doped samples.

\subsection{Gas Sensor}

All the polypyrrole samples were studied for detection of ammonia gas. A typical plot of current vs. time for polypyrrole prepared using $\mathrm{FeCl}_{3}$ as oxidant and exposed to ammonia gas is given in Figure 6. All samples were studied for 3 cycles to check their reproducibility and absorption and desorption process. It may be seen from the Figure 6 that the I vs time plot for $2^{\text {nd }}$ and $3^{\text {rd }}$ cycles somewhat differ from the first cycle. This may be because desorption may be not completed within the given time.

The Sensitivity factor is calculated using the equation

$$
S=\frac{R g-R o}{R o}
$$

where $R g$ and $R o$ are resistances with gas and without gas (in air) respectively $[17,18]$. The values calculated during the present investigation for sensors fabricated using differently doped polypyrrole and for different gases are given in Table 2.

The response of different materials towards ammonia gas was seen to be different. When pure Ppy and Ppy doped with $\mathrm{LiClO}_{4}$ was exposed to ammonia gas there was an increase in current. This behavior is exactly opposite to that observed for Ppy prepared by electrochemical method $[7,14]$. This is mainly because in chemical preparation $\mathrm{Cl}^{-}$ion is incorporated as counter ion to maintain the electroneutrality. Usually partial oxidation state (upto +0.33 ) is produced in the process. In addition it is observed that some amount of ions remain within the polypyrrole in the $\mathrm{Fe}^{2+}$ state. Thus there is a possibility that higher oxidation state can be induced in the interaction with ammonia. In such a situation the higher charge on cations can lead to increase in current. When $\mathrm{LiClO}_{4}$ was used as dopant the situation is same with additional $\mathrm{ClO}_{4}^{-}$ions interacting with pyrrole ring.

Table 2. Sensitivity for Ammonia of Ppy prepared with different dopants.

\begin{tabular}{cc}
\hline Sample & Sensitivity for Ammonia \\
\hline Pure Polypyrrole & 0.84 \\
Polypyrrole with $\mathrm{LiClO}_{4}$ & 0.86 \\
Polypyrrole with p-TS & 0.55 \\
Polypyrrole with NSA & 1.66 \\
\hline
\end{tabular}


<smiles>Cc1ccc(-c2ccc(-c3ccc(-c4ccc(-c5ccc(-c6ccc(C)[nH]6)[nH]5)[nH]4)[nH]3)[nH]2)[nH]1</smiles>

POLYPYRROLE (UNDOPED)

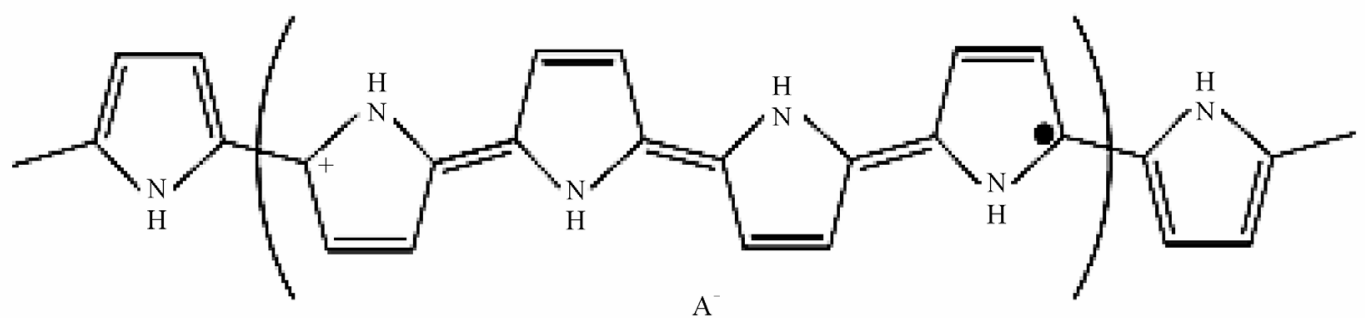

POLARON

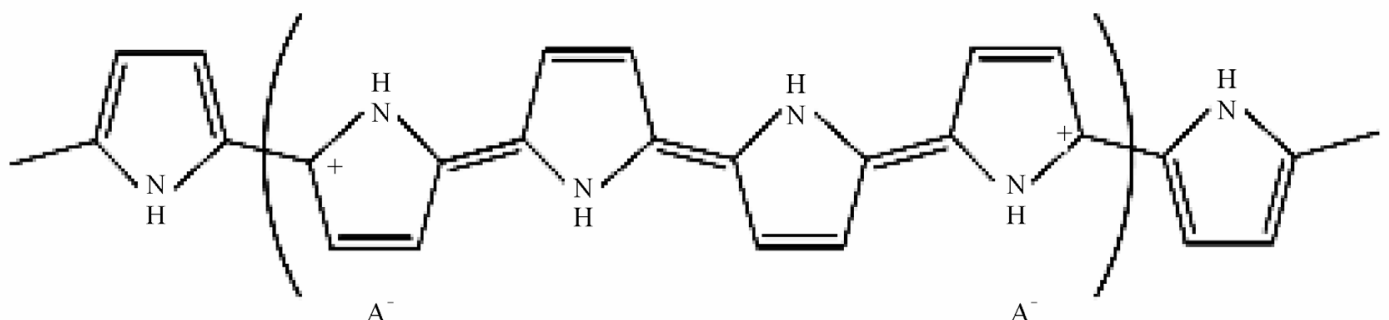

BIPOLARON

Conduction Band
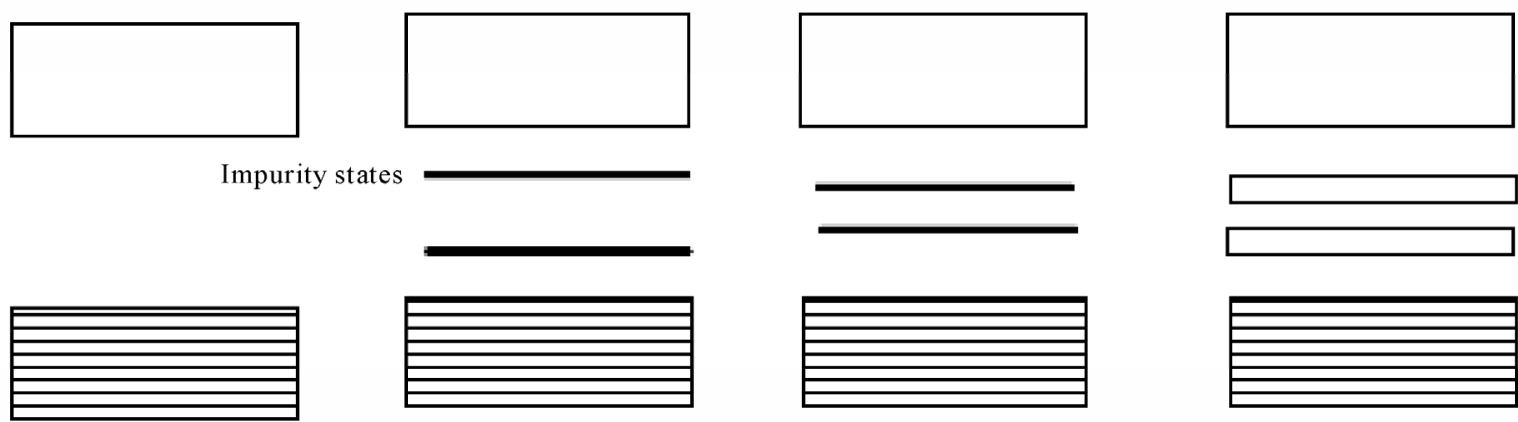

Valence Band

Polaron

Bipolalaron

Bipolalaron Band

Figure 5. Conduction mechanism in Polypyrrole.

Therefore the material doped with $\mathrm{LiClO}_{4}$ also show increase in current when exposed to ammonia gas.

It was noted during these investigations that when Ppy doped with p-TS and NSA were used, a decrease in current was observed when exposed to ammonia gas. The electrical conductivity of these two products shows higher conductivity than pure Ppy which means that the doping levels and the number of charge carriers produced in the process is quite high. This is probably due to the presence of sulfonic acid group inducing more 


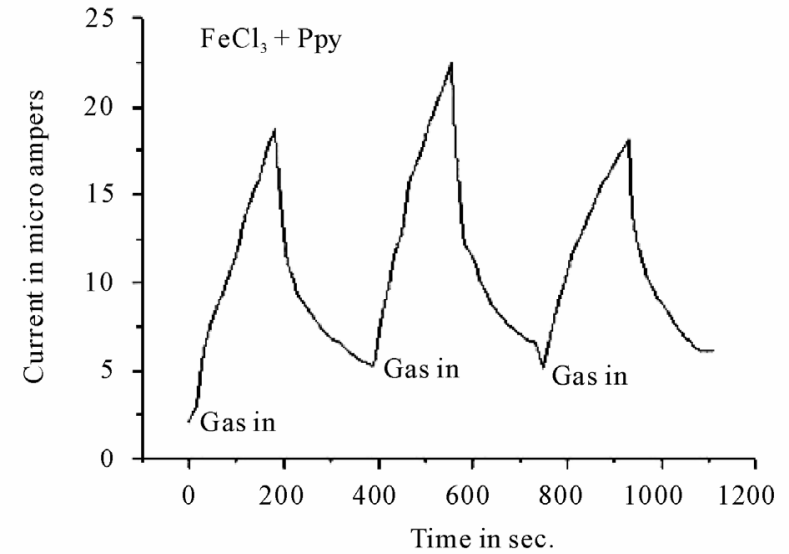

(a)

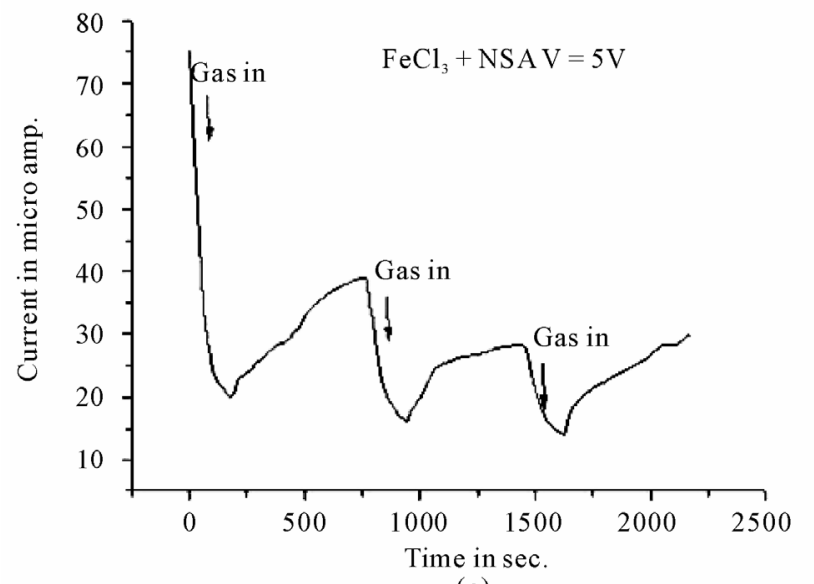

(c)

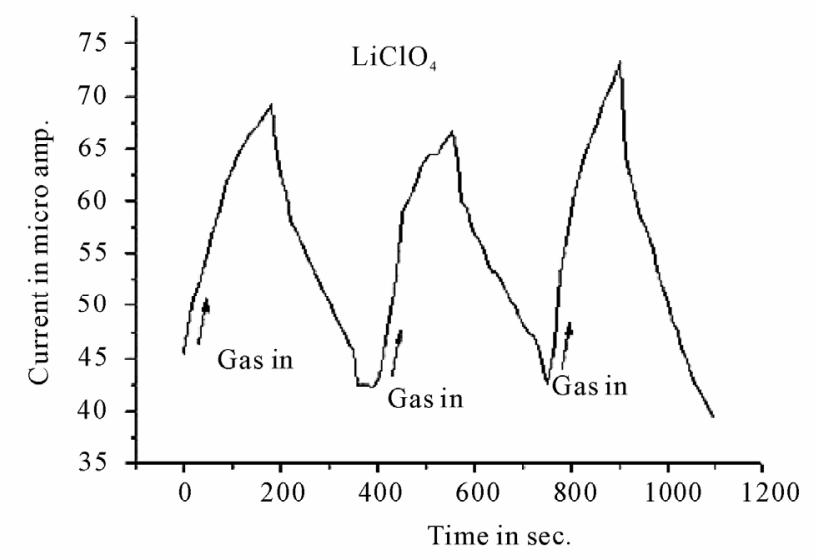

(b)

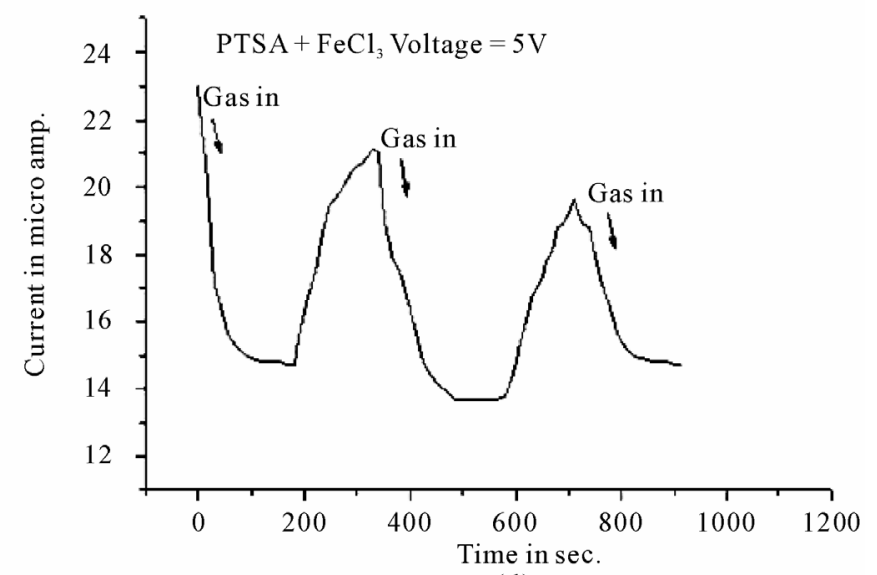

(d)

Figure 6. Gas sensing response for Ammonia gas of Polypyrrole prepared using (a) $\mathrm{FeCl}_{3}$ (b) $\mathrm{FeCl}_{3}+\mathrm{LiClO}_{4}(\mathrm{c}) \mathrm{FeCl}_{3}+\mathrm{NSA}$ (d) $\mathrm{FeCl}_{3}+$ P-TS.

charge on pyrrole ring. Thus there already exists a higher density of charge and therefore the presence of ammonia cannot produce more charges, but rather decrease the effective charge. In such a situation therefore the conductivity of Ppy will decrease, which has indeed been observed in our studies.

The four different samples produced in the present studies were also tested for their ability to detect LPG gas. It was found that when LPG gas was injected the electrical conductivity of pure Ppy was found to be decreasing as shown in Figure 7(a). However when the Ppy samples doped with p-TS and NSA were used it was observed that the electrical current increases to a small extent ( 1 to $2 \mu \mathrm{a}$ ) when exposed to LPG gas. This shows that the type of dopant has a great effect on the change in the electrical resistance. It is interesting to find that these changes are exactly in opposite direction to those observed with the experiments on ammonia gas. However the range of effective change in the currents were found to be quite small as compared to changes observed when sensors were exposed to ammonia gas. Further the re- sponse time for LPG was observed to be in terms of few minutes whereas with the ammonia gas it was just few seconds. LPG gas consist of Butane $\left(\mathrm{C}_{4} \mathrm{H}_{10}\right)$ and Propane $\left(\mathrm{C}_{3} \mathrm{H}_{8}\right)$ and as such are not much capable of electronic interaction with the polypyrrole. Thus it seems that Ppy materials prepared in the present investigation have very good capability of detection of ammonia but are not efficient for detection of LPG gas.

\section{Conclusions}

Polypyrrole was synthesized in the pure form and doped with $\mathrm{LiClO}_{4}, \mathrm{p}$-TS and NSA. All these four varieties were characterized using FTIR, X ray diffraction and SEM. It was seen that there are structural and morphological differences which affects their electrical properties. The electrical conductivity was highest for $\mathrm{p}$-TS doped polypyrrole $\left(5.1 \times 10^{-2} \mathrm{~S} / \mathrm{cm}\right)$. These materials were used as gas sensors for the detection of ammonia and LPG. It was found that where as ammonia could be detected efficiently. The detection of LPG was not very 


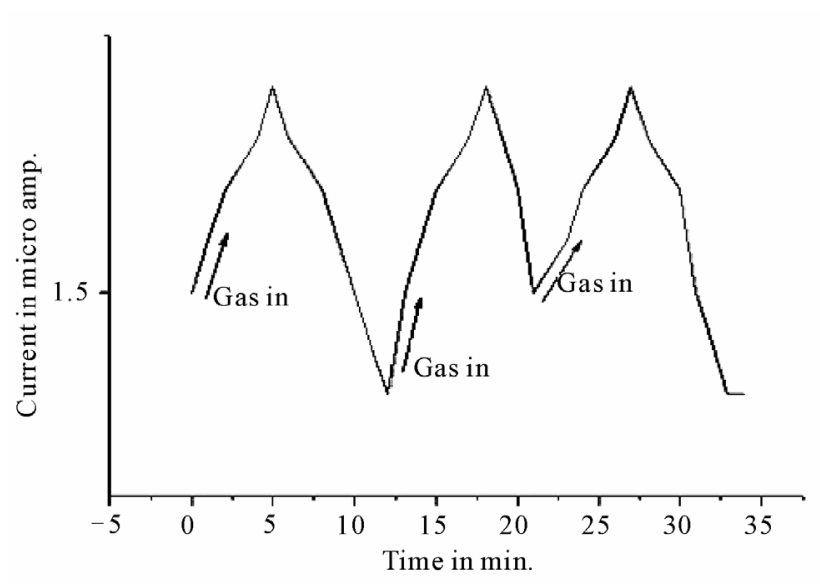

(a)

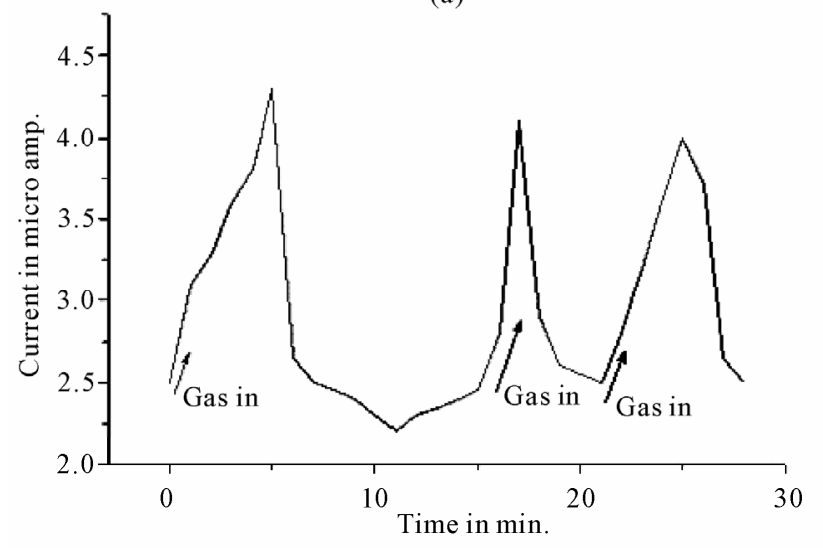

(c)

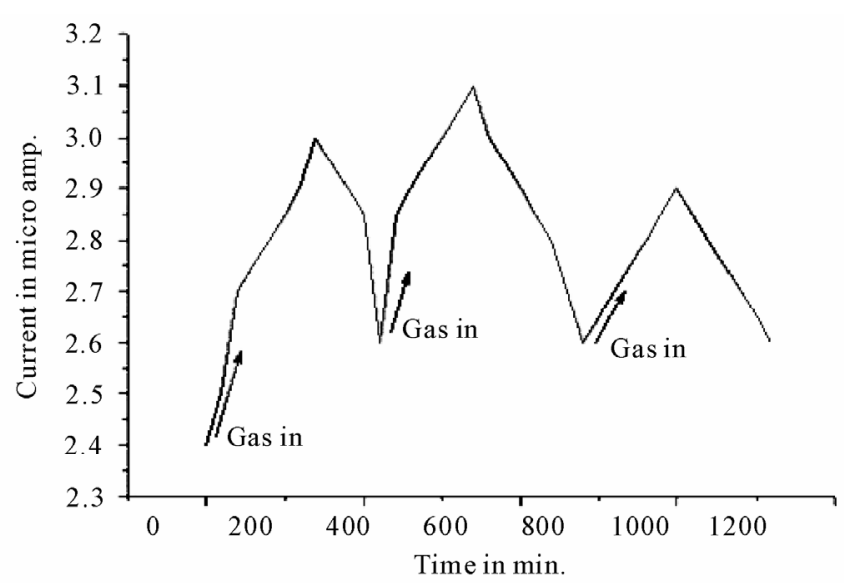

(b)

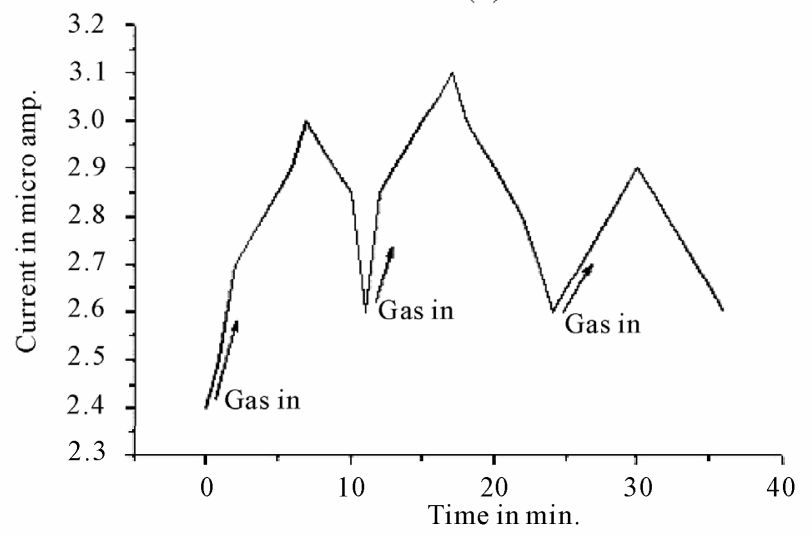

(d)

Figure 7. Gas sensing response for LPG (a) Ppy $+\mathrm{FeCl}_{3}$ (b) $\mathrm{Ppy}+\mathrm{LiClO}_{4}$, (c) Ppy + p-TS (d) Ppy + NSA.

promising.

\section{Acknowledgements}

The authors wish to thank Director, BTRA for interest and support during this work. Thank are also due to Mr. A.V. Gore for help during the experimental work. H.K.C. is grateful to the Principal Dnyanasadhana College for granting the necessary leave.

\section{References}

[1] S. C. Hernandez, D. Chaudhari, W. Chen, N. Myung and A. Mulchandani, "Single Polypyrrole Nanowire Ammonia Gas sensor," InterScience, Vol. 19, No. 19-20, 2007, pp. 2125-2130.

[2] S. Cosnier, C. Gondran, R. Wessel, F. T. Montforts and M. Wedel. "A Poly (Pyrrole - Cobalt (II) Deuteroporphyrin) Electrode for the Potentiometric Determination of Nitrite," Sensors, Vol. 3, No. 7, 2003, pp. 213-222. doi:10.3390/s30700213

[3] M. F. Mabrook, C. Pearson and M. C. Petty, "Inkjet Printed Polypyrrole Thin Films for Vapor Sensing," Sensors and Actuators B: Chemical, Vol. 115, No. 1, 2006, pp. 547-557.

[4] R. A. Bissell, K. C. Persaud and P. Travers, "The Influence of Non-Specific Molecular Partitioning of Analytes on the Electrical Response of Conducting Organic Polymer Gas Sensors," Physical Chemistry Chemical Physics, Vol. 4, No. 14, 2002, pp. 3482-3490. doi: $10.1039 / \mathrm{b} 201292 \mathrm{~h}$

[5] H. Bai and G. Shi, "Gas Sensors on Conducting Polymers," Sensors, Vol. 7, No. 3, 2007, pp. 267-307. doi:10.3390/s7030267

[6] L. A. Mashat, H. D. Tran, W. Wlodarski, R. B. Kaner and K. K. Zadeh, "Conductometric Hydrogen Gas Sensors Based on Polypyrrole Nanofibers," IEEE Sensors, Vol. 8, No. 4, 2008, pp. 365-370.

[7] A. Joshi, S. A. Gangal, N. Padma, D. K. Aswal and S. K. Gupta, "Gas Sensing Properties of Polypyrrole Thin Films," 2008.

http://www.barc.ernet.in/publications/nl/2008/20081038. pdf

[8] S. Hosein and A. A. Entezami, "Polypyrrole Based Toxic Gas Sensors by Mass and Conductivity Measurements," Iranian Polymer Journal, Vol. 8, No. 3, 1999, pp. 205213.

[9] H. Yoon, M, Chang and J. Jang, "Sensing Behavior of 
Polypyrrole Nano Tubes Prepared in Reverse Micromulsions: Effects of Tranducers Size and Transduction Mechanism," Journal of Physical Chemistry B, Vol. 110, No. 29, 2006, pp. 14074-14077. doi:10.1021/jp061423b

[10] V. Saxena, S. Choudhary, S. C. Gadkari, S. K. Gupta and J. V. Yakhmi, "Room Temperature Operated Ammonia Gas Sensor Using Polycarbazole Langumuir-Blodgett film," Sensors and Actuators B: Chemical, Vol. 107, No. 1, 2005, pp. 277-282. doi:10.1016/j.snb.2004.10.011

[11] L. Jiang, H. K. Jun, Y. S. Hoh, J. O. Lim, D. D. Lee and J. S. Huh, "Sensing Characteristics of Polypyrrole - Poly (Vinyl Alcohol) Methanol Sensors Prepared by in Situ Vapour State Polymerization," Sensors and Actuators B: Chimical, Vol. 105, No. 2, 2005, pp. 132-137. doi:10.1016/j.snb.2003.12.077

[12] R. Ansari, "Polypyrrole Conducting Electro Active Polymers: Synthesis and Stability Studies," E-Journal of Chemistry, Vol. 3, No. 13, 2006, pp. 186-201.

[13] H. Kato, O. Nishikwa, T. Matsui, S. Honma and H. Kokado, "Fourier Transform Infrared Spectroscopy Study of Conducting Polymer Polypyrrole Higher Order Structure of Electrochemically Synthesized Film," The Journal of Physical Chemistry, Vol. 95, No. 15, 1991, pp. 60146016. doi:10.1021/j100168a055
[14] N. V. Bhat, A. P. Gadre and V. A. Bambole, "Structural, Mechanical and Electrical Properties of Electro polymerized Polypyrrole Composite Films," Journal of Applied Polymer Science, Vol. 80, No. 13, 2001, pp. 2511-2517, doi:10.1002/app.1359

[15] T. K. Vishnuvardhan, V. R. Kulkarni, C. Basavaraja and S. C. Raghavendra, "Synthesis, Characterization and A. C. Conductivity of Polypyrrole $/ \mathrm{Y}_{2} \mathrm{O}_{3}$ Composites," Material Science, Vol. 29, No. 1, 2006, pp. 77-83.

[16] H. Eisazadeh, "Studying the Characteristics of Polypyrrole and Its Composites," World Journal of Chemistry, Vol. 2, No. 2, 2007, pp. 67-74

[17] R. Turcu, M. Brie, G. Leising, V. Tosa, A. Mihut, A. Niko and A. Bot, "FTIR Reflectance Studies of Electrochemically Prepared Polypyrrole Films," Applied Physics A, Vol. 67, No. 3, 1998, pp. 283-287. doi:10.1007/s003390050772

[18] S. A. Waghule, S. M. Yenorkar, S. S. Yawale and S. P. Yawale, " $\mathrm{SnO}_{2} / \mathrm{PPy}$ Screen - Printed Multilayer $\mathrm{CO}_{2} \mathrm{Gas}$ Sensor," Sensors and Transducers, Vol. 79, No. 5, 2007, pp. 1180-1185. 